\title{
Pre-existing Schistosoma japonicum infection alters the immune response to Plasmodium berghei infection in C57BL/6 mice
}

\author{
Mei-lian Wang ${ }^{1}$, Ya-ming Cao ${ }^{2}$, En-jie Luo ${ }^{1 *}$, Ying Zhang ${ }^{3}$ and Ya-jun Guo ${ }^{3}$
}

\begin{abstract}
Background: Since helminths and malaria parasites are often co-endemic, it is important to clarify the immunoregulatory mechanism that occurs during the process of co-infection. A previous study confirmed that dendritic cells (DCs) are involved in the establishment and regulation of the T-cell-mediated immune response to malaria infection. In the current study, distinct response profiles for splenic DCs and regulatory T cell (Treg) responses were assessed to evaluate the effects of a pre-existing Schistosoma japonicum infection on malaria infection.
\end{abstract}

Methods: Malaria parasitaemia, survival rate, brain histopathology and clinical experimental cerebral malaria (ECM) were assessed in both Plasmodium berghei ANKA-mono-infected and S. japonicum-P. berghei ANKA-co-infected mice. Cell surface/intracellular staining and flow cytometry were used to analyse the level of splenic DC subpopulations, toll-like receptors (TLRs), DC surface molecules, Tregs $\left(C D 4^{+} \mathrm{CD} 25^{+} \mathrm{Foxp} 3^{+}\right)$, IFN- $\gamma / \mathrm{IL}-10$-secreting Tregs, and IFN- $\gamma^{+} / \mathrm{IL}-10^{+}-\mathrm{Foxp3}^{-} \mathrm{CD} 4^{+} \mathrm{T}$ cells. IFN-Y, IL-4, IL-5, IL-10 and IL-13 levels were determined in splenocyte supernatants using enzyme-linked immunosorbent assay (ELISA).

Results: The co-infected mice had significantly higher malaria parasitaemia, compared with the mono-infected mice, on days 2, 3, 7 and 8 after $P$. berghei ANKA infection. Mono-infected mice had a slightly lower survival rate, while clinical ECM symptoms, and brain pathology, were significantly more severe during the period of susceptibility to ECM. On days 5 and 8 post $P$. berghei ANKA infection, co-infected mice had significantly lower


IFN- $\gamma$-secreting Tregs, and IFN- $\gamma^{+}{ }^{+} \operatorname{Poxp}^{-} \mathrm{CD}^{+} \mathrm{T}$ cells in single-cell suspensions of splenocytes when compared with $P$. berghei ANKA-mono-infected mice. Co-infected mice also had significantly lower levels of IFN- $y$ and higher levels of IL-4, IL-5, and IL-13 in splenocyte supernatants compared to mono-infected mice. There were no differences in the levels of IL-10-secreting Tregs or IL-10 ${ }^{+} \mathrm{Foxp}^{-} \mathrm{CD}^{+} \mathrm{T}$ cells between co-infected and mono-infected mice.

Conclusions: A Tregs-associated Th2 response plays an important role in protecting against ECM pathology. Pre-existing S. japonicum infection suppressed TLR ligand-induced DC maturation and had an anti-inflammatory effect during malaria infection not only by virtue of its ability to induce Th2 responses, but also by directly suppressing the ability of DC to produce pro-inflammatory mediators.

Keywords: Plasmodium berghei ANKA, Schistosoma japonicum, Co-infection, Inflammatory cytokine

\footnotetext{
* Correspondence: enjieluo@hotmail.com

'Department of Microbiology and Parasitology, College of Basic Medical Sciences, China Medical University, No. 92 Beier Road, Heping District, Shenyang 110001, China

Full list of author information is available at the end of the article
} 


\section{Background}

Malaria is an infectious disease caused by the Plasmodium parasite that continues to be a health issue for humans. It is one of the most common pathogenic factors of morbidity and mortality in sub-Saharan Africa [1]. More than one million children are dying each year as a result of malaria infection [2]. Experimental cerebral malaria (ECM), caused by infection with Plasmodium berghei, can result in cerebral inflammation and is a form of malaria that is life threatening in humans. The prominent pathogenesis of cerebral malaria (CM) is adherence and sequestration of parasitized red blood cells ( $\mathrm{pRBCs}$ ), immune cells and platelets to the vascular endothelial cells lining the small blood vessels of the brain. This leads to microhaemorrhages and oedema in the brain $[3,4]$.

Immunity to malaria has been studied extensively, however it is still not fully understood. It is generally thought that the balance between pro- and anti-inflammatory cytokines plays a very important role in the regulation of the immune response and pathogenesis [5]. A strong Th1 immune response to intracellular Plasmodium could prevent multiplication by this organism during the early stages of malaria infection, thus impacting the course of the disease. However, the body may then be exposed to severe immunopathology due to excessive production of pro-inflammatory cytokines (e g, interferon- $\gamma$, IFN- $\gamma$ ), combined with inadequate production of others (e g, interleukin-10, IL-10), constituting a passive effect. It is possible though, that the course of Plasmodium infection could be changed, if the balance of pro- and antiinflammatory cytokines were broken, such as with a concomitant helminth infection.

Schistosome infections are common in many tropical regions of the world, ranking second only to malaria $[4,6]$. Three main Schistosoma species, Schistosoma mansoni, Schistosoma japonicum, and Schistosoma haematobium [7], frequently infect humans and these infections significantly impact public health. Recently, there has been an increasing awareness that helminth infections can ameliorate pro-inflammatory conditions due to their inherent ability to induce Th2 responses to various cytokines and pathways [8,9]. Kane et al. demonstrated that helminths had direct anti-inflammatory effects on innate immune responses. In that study, it was reported that the eggs of $S$. mansoni could suppress LPS-induced activation of immature murine dendritic cells (DCs) [10].

As helminths and malaria parasites are often coendemic, schistosomiasis and malaria are frequently observed concomitantly. The existence of shared antigens and cross-reactive antibodies to different components of the two parasites has been confirmed in a previous report [6]. In the past few years more and more studies have been conducted to elucidate the immune mechanism(s) involved in worm and malaria co-infections. However, many of these studies have produced conflicting results, which has made it difficult to clearly understand the outcomes of these co-infections [11]. Some studies have reported an increased incidence of falciparum malaria in hosts with $S$. mansoni [12] while other studies have indicated that $S$. haematobium provides some protection from malaria (e g, lower parasitaemia, and lower incidence) $[13,14]$. These contradictory results may be caused by differences between different helminths and the infection stage of the parasites [5,15-17]. Currently, $S$. mansoni is the most widely used schistosome species for evaluating host immune responses [18]. To date, no reports on co-infections with S. japonicum and Plasmodium have been found. According to a previous report, S. japonicum infection was associated with more severe hepatic disease in humans than compared with $S$. mansoni infection [19]. It was suggested that there was a significant immunological difference between S. japonicum and S. mansoni [19].

In the present study, $S$. japonicum, along with the $P$. berghei ANKA strain were used to produce a coinfection model in C57BL/6 mice. This model is likely a better fit for investigating the immunomodulatory mechanism of this co-infection in Southern Chinese populations since $S$. japonicum is the only schistosome species present in South China.

It has previously been demonstrated that Tregs can suppress Th1 responses to malaria infection by modifying DCs [20]. In the current study, pre-existing $S$. japonicum infection strengthened the Tregs-associated Th2 response to the malaria infection and this Th2 response played an important role in protecting against ECM pathology. In addition, S. japonicum infection suppressed the proliferation of DC subpopulations and weakened DC maturation and cytokine secretion. This indicated that pre-existing S. japonicum infection had anti-inflammatory effects during the malaria infection, not only by virtue of its ability to induce Th2 responses, but also by directly suppressing the ability of DCs to produce pro-inflammatory mediators.

\section{Methods}

\section{Mice, parasites, and experimental infection}

Female C57BL/6 mice, four weeks old, were purchased from the Beijing Animal Institute (Beijing, China). They were kept in the animal facility at China Medical University. Mice were maintained in individually ventilated cages and supplied with heat-sterilized food and distilled water ad libitum. The mice were randomly assigned to three groups. 25 mice were infected with $P$. berghei ANKA ( $P$. berghei ANKA-mono-infection group), 15 mice were infected with $S$. japonicum. ( $S$. japonicum-mono-infection group), and 25 mice were infected with both S. japonicum and P. berghei ANKA (co-infection group). 
The S. japonicum strain was obtained from the Jiangsu Institute of Parasitic Diseases (Wuxi, China). A total of 50 cercariae of S. japonicum were administered percutaneously to $\mathrm{C} 57 \mathrm{BL} / 6$ mice when the mice were five weeks of age.

Plasmodium berghei ANKA strain was provided by Dr Motomi Torii (Department of Molecular Parasitology, Ehime University Graduate School of Medicine, Ehime, Japan). Parasites were stored as frozen stabilates at $-80^{\circ} \mathrm{C}$. To obtain experimental inocula of $P$. berghei ANKA, pRBCs were sequentially passaged through three homologous donor mice. Infections were initiated in C57BL/6 mice by intraperitoneal (ip) injection of $1 \times 10^{6} P$. berghei ANKA-pRBCs eight weeks after infection with $S$. japonicum.

Three mice each from the $P$. berghei ANKA-mono-infection group, the S. japonicum-mono-infection group, and the co-infection group were euthanized at $0,3,5$, and 8 day post- $P$. berghei ANKA infection.

The current study has been reviewed and approved by China Medical University Institute of Medical Research Animal Ethics Committee.

\section{Confirmation of helminth infection}

Helminth infection was confirmed by the presence of worms and liver granulomas upon necropsy. Worms were obtained by portal perfusion [21], and livers were examined for the presence of granulomas under a stereomicroscope.

\section{Malaria parasitaemia, survival rates and disease assessment}

Parasitaemia was determined by light microscopy of Giemsa-stained, thin (tail) blood smears. Slides were coded and pRBCs were counted microscopically in at least five microscopic fields, each containing approximately 300 cells.

Mice were monitored for mortality daily, post- $P$. berghei ANKA infection, to evaluate the survival rate of $P$. berghei ANKA-mono-infected and the co-infected mice. Clinical ECM was also assessed and was defined by the presence of the following signs [22]: ruffled fur, hunching, wobbly gait, limb paralysis, convulsions, and coma. Each sign was given a score of 1 . Animals with scores $\geq 4$ were considered to have severe ECM.

\section{Spleen cell culture}

Spleen cell culture was prepared as previously described $[22,23]$. Briefly, the spleen was aseptically removed from each mouse and pressed through a sterile fine-wire mesh with $10 \mathrm{ml}$ of RPMI-1640 (Life Technologies, Shanghai, China) supplemented with $5 \%$ heat-inactivated fetal calf serum (FCS; Hyclone Laboratories, Inc, South Logan, Utah, USA), 25 mM Hepes (Life Technologies, Shanghai, China), $0.12 \%$ gentamicin (Schering-Plough, Kenny Worth, New
Jersey, USA), and $2 \mathrm{mM}$ glutamine (Life Technologies, Shanghai, China). Cell suspensions were centrifuged at 350 $\mathrm{g}$ for $10 \mathrm{~min}$ at room temperature (RT). Erythrocytes were lysed with cold $0.17 \mathrm{M} \mathrm{NH}{ }_{4} \mathrm{Cl}$ and the cells were washed twice with fresh medium. The viability of the spleen cells was determined by trypan blue exclusion and was always $>90 \%$. Spleen cells were adjusted to a final concentration of $10^{7}$ cells/ml in RPMI-1640 supplemented with $10 \%$ heat-inactivated FCS. Aliquots (500 $\mu \mathrm{l} /$ well) of the cell suspension were incubated in 24-well flat bottom culture plates (Falcon ${ }^{\circ}$, Corning Life Sciences, CA, USA) in triplicate for $48 \mathrm{hr}$ at $37^{\circ} \mathrm{C}$ in a humidified $5 \% \mathrm{CO}_{2}$ incubator. The 24-well plates were then centrifuged at 350 $\mathrm{g}$ for $10 \mathrm{~min}$ at RT and the supernatants were collected and stored at $-80^{\circ} \mathrm{C}$ until assayed for cytokine levels.

\section{Cytokine analysis}

IFN- $\gamma$, IL-4, IL-5, IL-10 and IL-13 levels in splenocyte supernatants were measured using commercial enzymelinked immunosorbent assay (ELISA) kits according to the manufacturer's protocol (R\&D Systems, Minneapolis, $\mathrm{MN}$, USA). The OD values were read in a microplate reader at $450 \mathrm{~nm}$. The concentrations of cytokines in samples were calculated against the standard curve generated using recombinant cytokines.

\section{Cell surface/intracytoplasmic staining and flow cytometry}

All flow cytometry analyses were performed on a FACS Calibur (BD Biosciences, San Diego, CA, USA) and analysed with Cell Quest software (version 3.3; BD Biosciences, CA, USA). All antibodies for FACS were purchased from BD Biosciences or e Bioscience, unless otherwise indicated.

For analysis of spleen DCs, single-cell suspensions of splenocytes were first pre-incubated with anti-mouse CD16/32 (2.4G2) monoclone antibody (mAb) to block Fc receptors and then stained with a combination of FITC-conjugated anti-mouse CD11c (clone HL3) $\mathrm{mAb}$, PE-conjugated anti-mouse CD11b (clone M1/70) mAb, PerCP-conjugated anti-mouse CD45R/B220 (clone RA3-6B2) mAb, PE-conjugated anti-mouse MHC class II (clone M5/115.15.2) mAb, and PE-conjugated anti-mouse CD86 (clone GL1) mAb. Isotype-matched Abs were used as staining controls.

DC cell surface TLRs were also evaluated. For TLR4 analysis, splenocytes were harvested, blocked with antiCD16 / CD32, and then stained using a combination of FITC-conjugated anti-mouse CD11c (clone HL3) and PE-conjugated anti-mouse TLR4 (MTS510).

For intracellular TLR9 staining of DCs, splenocytes were blocked with anti-CD16CD32 after harvesting and then stained using FITC-conjugated anti-mouse CD11c (clone HL3). After fixation and permeabilization using staining buffer reagents as instructed by the manufacturer, cells 
were incubated with biotinylated anti-mouse TLR9 (clone 5G5, Hycult Biotechnology (HBT), Uden, The Netherlands) followed by PE-conjugated streptavidin (Biolegend, San Diego, CA, USA).

Spleen cells that were previously collected from C57BL/ 6 mice at different time points after infection and stimulated with ConA (5-10 ug/ml) for $48 \mathrm{hr}$ and trypsinized during the final $8 \mathrm{hr}$ were used to analyse Tregs and IL10 -secreting Tregs in the spleen $\mathrm{CD}^{+} \mathrm{T}$ cell population and to analyse the IL-10 levels produced by Foxp $3^{-} \mathrm{CD} 4^{+} \mathrm{T}$ cells. Cell concentration was then adjusted to $2 \times 10^{6} / \mathrm{ml}$, followed by a $4 \mathrm{hr}$ stimulation with plate-bound antimouse CD3 $(1 \mu \mathrm{g} / \mathrm{ml})$ and anti-mouse CD28 $(0.2 \mu \mathrm{g} / \mathrm{ml})$, combined with Golgi Stop (Cat no 554724). After continued co-culture at $37^{\circ} \mathrm{C}$ for $4 \mathrm{hr}$, cells were washed with $3 \%$ FCS and re-suspended in $100 \mu \mathrm{l}$ of $3 \%$ FCS. FITCconjugated anti-mouse CD4 and PE- conjugated antimouse CD25 (clone 3C7) were added for surface staining. The cells were then fixed, permeabilized, and intracytoplasmic staining was performed using APC-conjugated anti-Foxp3 (clone FJK16s) and PerCP-Cy5.5-conjugated anti-IL-10 (clone JES5-16E3). FITC-conjugated rat IgG2b was used as the isotype control.

To analyse IFN- $\gamma$-secreting Tregs in the spleen $\mathrm{CD}^{+} \mathrm{T}$ cell population and to analyse IFN- $\gamma$ produced by Foxp $3^{-}$ $\mathrm{CD} 4^{+} \mathrm{T}$ cells, spleen cells, that had been previously collected from C57BL/6 mice at different time points after infection and stimulated with PMA and ionomycin for 2 hr at $37^{\circ} \mathrm{C}$ and then Golgi Stop (Cat no 554724), were added to each reaction $(1: 500[\mathrm{v} / \mathrm{v}])$. The next steps (coculture, washing with FCS, re-suspending, surface staining with FITC-conjugated anti-CD4 and PE- conjugated antiCD25) were carried out as described above. The cells were then fixed, permeabilized, and intracytoplasmic staining was performed using APC-conjugated anti- Foxp3 (clone FJK16s) and PerCP-Cy5.5-conjugated anti-IFN- $\gamma$. FITCconjugated rat IgG2b was used as the isotype control.

\section{Histopathology}

Immediately after death, the brains of the mice were removed and fixed in 1\% buffered formalin for $48 \mathrm{hr}$. The brain tissue was then dehydrated using graded alcohols and xylene, and then embedded in paraffin. Continuous coronal sections of the tissue were made using a microtome. Five randomly selected sections were made into slides and stained with hemalaun eosin. The haemorrhage and the infiltration of immune cells were then examined in sections from the co-infected, $P$. berghei ANKA -mono-infected, and the normal control mice.

\section{Statistical analysis}

Data were analysed using Prism (GraphPad, La Jolla, CA). Independent-samples $t$-tests were performed. All tests were considered significant when $P<0.05$.


C

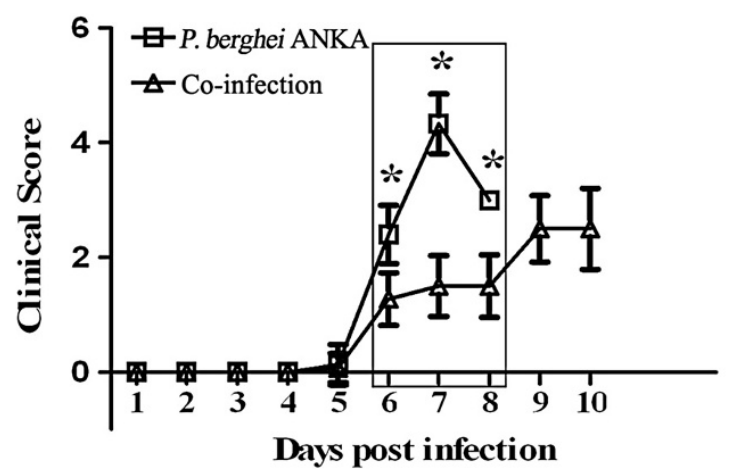

Figure 1 Parasitaemia (A), survival rate (B), and clinical disease assessment by clinical score (C) in C57BL/6 mice infected with Schistosoma japonicum and then inoculated with $1 \times 10^{6}$ Plasmodium berghei ANKA-pRBCs eight weeks later (co-infection) and were analysed together with $P$. berghei ANKA-mono-infected mice ( $P$. berghei ANKA). For monitoring the malaria parasitaemia, a Giemsa-stained thin smear was made daily. The open box indicates the susceptible period for $\mathrm{CM}$. Values represent the means with SD ( $n=3$ mice per group). Student $t$ test by comparisons between the two groups was performed with * indicating $P<0.01$ 


\section{Results}

Parasitaemia, survival rate and disease assessment of ECM

Malaria parasitaemia (Figure 1A), mortality (Figure 1B) and ECM scores (Figure $1 C$ ) were recorded daily in the $P$. berghei ANKA-mono-infection mice and the coinfection group mice and comparisons of these were made between the 2 groups. During the challenge, the mean parasitaemia of the mice in both groups increased except for a transient decline that was observed on day 7 post-infection (pi). The co-infected mice had a significantly higher level of parasitaemia than $P$. berghei ANKA-mono-infected mice on day 2, 3, 7 and 8 pi. Between day 6 and day 8 (the period of susceptibility to
CM), most of the mice presented clinical signs of ECM and subsequently died. Mice in the P. berghei ANKA group had significantly higher scores, based on the clinical scores used for assessment of clinical ECM symptoms, than mice in the co-infection group on day 6,7 , and 8 . This indicated that $P$. berghei ANKA-mono-infected mice had more severe cerebral pathology than did the co-infected mice. The survival rate of mice in the co-infection group was slightly higher than that in the $P$. berghei ANKA mono-infection group during the period of susceptibility to CM, but this was not significant. All animals died on day 9 and day 11 for $P$. berghei ANKA-mono-infected and the co-infected group, respectively.


C

$\mathrm{D}$
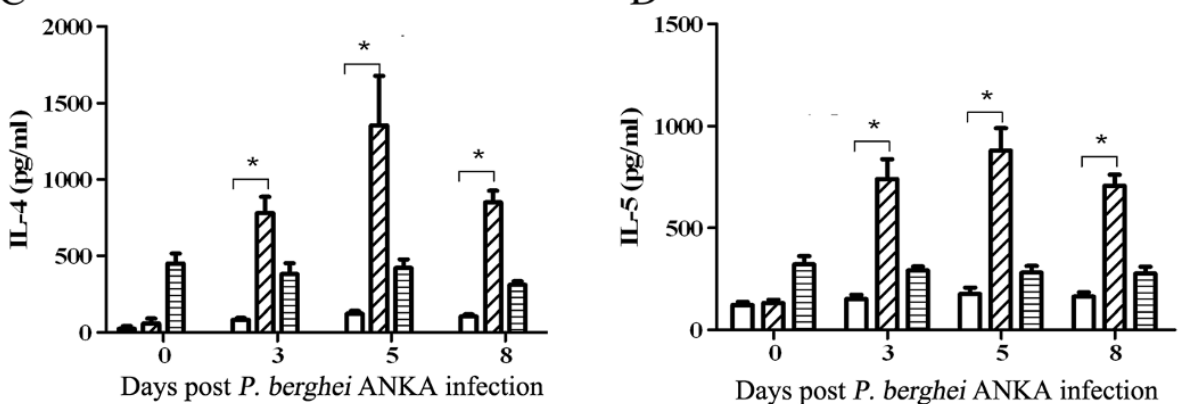

$\mathrm{E}$
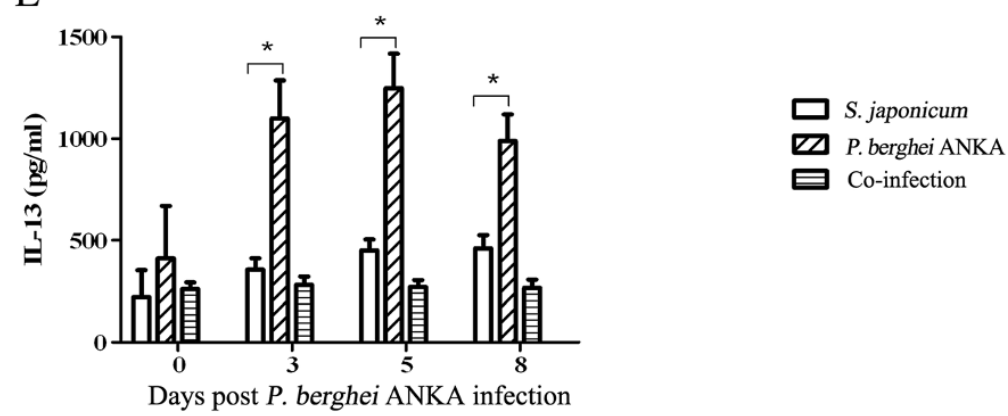

回-infection

Figure 2 C57BL/ 6 mice infected with Schistosoma japonicum and then inoculated with $1 \times 10^{6}$ Plasmodium berghei ANKA-pRBCs eight weeks later (co-infection) and were analysed together with $P$. berghei ANKA-mono-infected mice $(P$. berghei ANKA). The mice were dissected on days $0,3,5,8$ post $P$. berghei ANKA infection and splenocytes were cultured. Cytokines in the splenocyte culture supernatants were measured in duplicated wells using ELISA kits (R\&D Systems, Minneapolis, MN, USA) according to the manufacturer's instructions. The bar charts represent the level of splenic IFN-Y (A), IL-10 (B), IL-4 (C), IL-5 (D), and IL-13 (E) from at least three mice per group. Bars represent the mean values \pm SD. Student $t$ test by comparisons between the two groups was performed with * indicating $P<0.01$. 

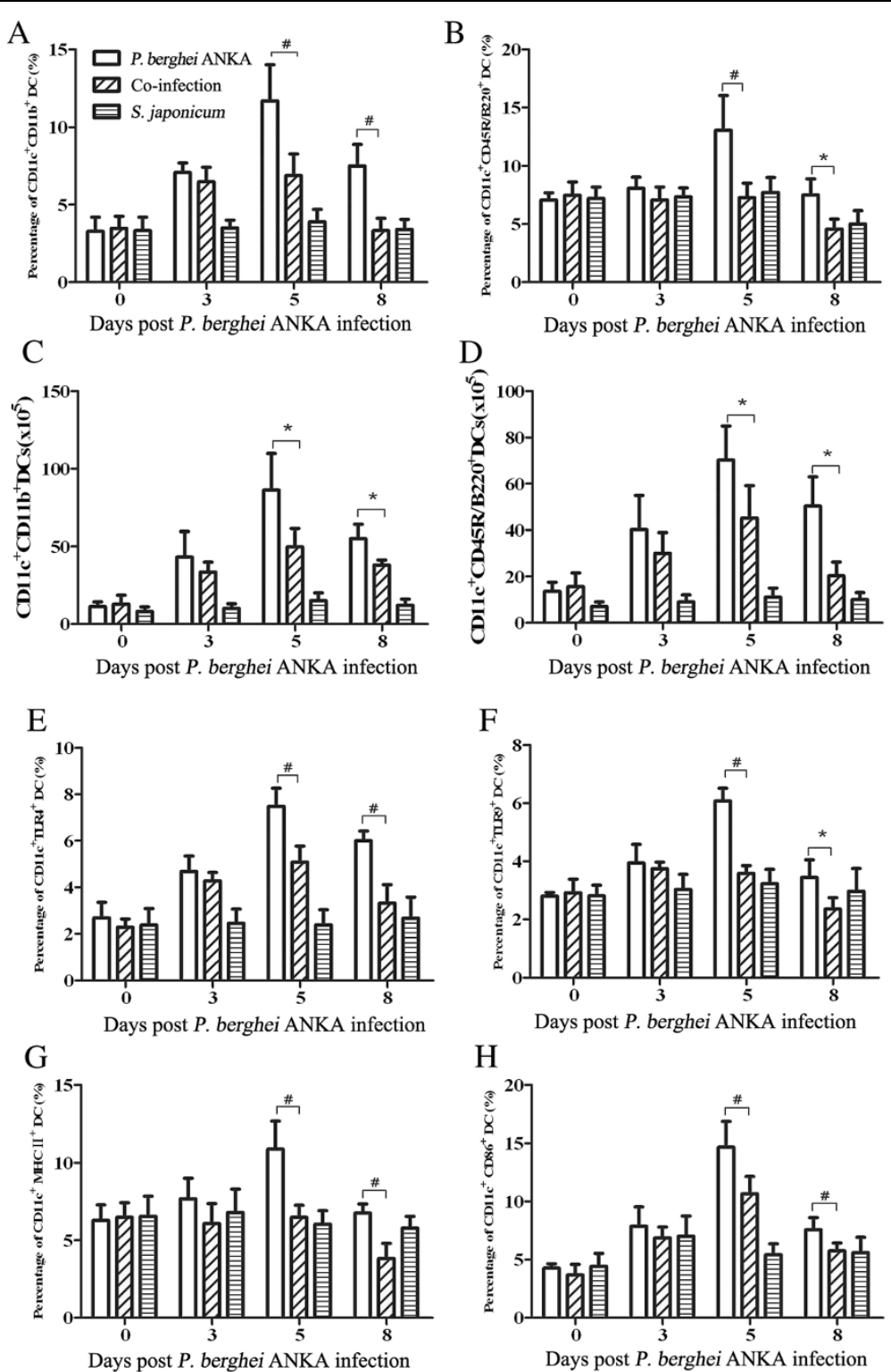

$\mathrm{H}$



I

$\mathrm{J}$

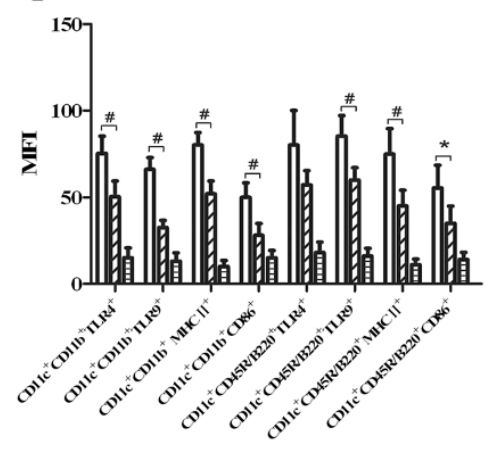

Day 5 post $P$. berghei ANKA infection

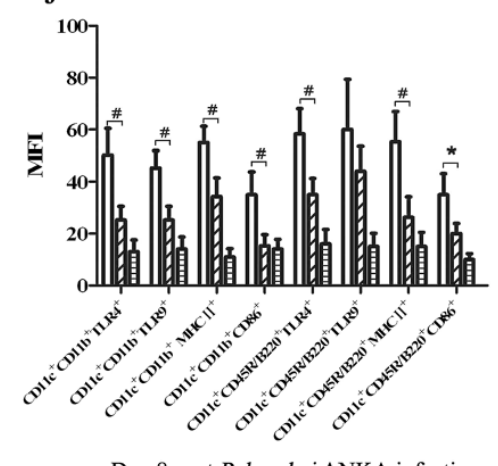

Day 8 post $P$. berghei ANKA infection 
(See figure on previous page.)

Figure 3 Mice in Plasmodium berghei ANKA-mono-infection group and Schistosoma japonicum-P. berghei ANKA-co-infection group were compared at different time points according to data obtained by flow cytometry. Proportion of splenic DCs subpopulation,

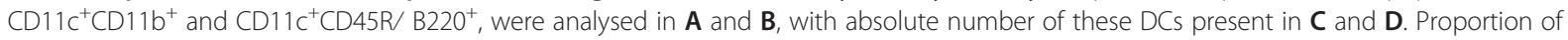
splenic CD11 $\mathrm{C}^{+}$DCs expressing TLR4, TLR9, MHC class II, and CD86 of the two groups were compared in $\mathbf{E}, \mathbf{F}, \mathbf{G}, \mathbf{H}, \mathbf{r e s p e c t i v e l y . ~ M F I ~ o f ~ T L R 4 , ~}$ TLR9, MHC class II, and CD86, which were expressed on CD11 $\mathrm{C}^{+}$CD $11 \mathrm{~b}^{+}$DCs and CD11 $\mathrm{C}^{+}$CD45R/ B220 $0^{+}$DCs were present in I and $\mathbf{J}$. At each time point, at least three mice per group were sacrificed. Bars represent the mean values \pm SD. Student $t$ test by comparisons between the two groups was performed with * indicating $P<0.05$ and \# indicating $P<0.01$.

All mice in the S. japonicum-mono-infected group survived until day 11 .

\section{Cytokine concentrations}

As this study aims to evaluate the effect of a pre-existing $S$. japonicum infection on a following malaria infection, changes in corresponding cytokines were monitored on days $0,3,5$ and 8 , post- $P$. berghei ANKA infection, in the co-infected, P. berghei ANKA-mono-infected, and in the $S$. japonicum-mono-infected mice. Because the mice in the $S$. japonicum-mono-infected group were not received any other treatments during this period, no changes were observed in the levels of the cytokines that were measured. Data from S. japonicum-mono-infected mice are presented in the following diagrams, however data was only compared between co-infected and P. berghei ANKA-mono-infected mice.

One pro-inflammatory cytokine (IFN- $\gamma$ ) and four anti-inflammatory cytokines (IL-4, IL-5, IL-10, and IL13) that were present in the supernatants of cultured splenocytes were measured by ELISA assay. This was done to evaluate the relationship between the levels of pro- and anti-inflammatory cytokines in the coinfected and P. berghei ANKA-mono-infected mice and to compare the levels between the two groups. Both the pro-inflammatory cytokine and the anti-inflammatory cytokines began to increase post- $P$. berghei ANKA infection, peaking on day 5 pi and then declining on day 8 pi. Compared with $P$. berghei ANKA-mono-infection group, the co-infection group showed a significantly lower level of IFN- $\gamma$ on day 5 and day 8 pi and significantly higher levels of IL-4, IL-5 and IL-13 on day 3, 5, 8 pi. No difference in the level of IL-10 was found when comparing the two groups (Figure 2).

DC subpopulations were defined as $C D 11 c^{+} C D 11 b^{+}$ and $\mathrm{CD} 11 \mathrm{c}^{+} \mathrm{CD} 45 \mathrm{R} / \mathrm{B} 220^{+}$cells by flow cytometry and the change in subpopulations of splenic DCs in the coinfected mice $v s$ P. berghei ANKA mono-infected mice were compared. Both the proportion/absolute cell numbers of $\mathrm{CD} 11 \mathrm{c}^{+} \mathrm{CD} 11 \mathrm{~b}^{+}$and $\mathrm{CD} 11 \mathrm{c}^{+} \mathrm{CD} 45 \mathrm{R} / \mathrm{B} 220^{+}$ began to increase post- $P$. berghei ANKA infection, peaking on day 5 pi. and then declining on day 8 pi. The co-infected mice had significantly lower percentages/cell numbers of both $\mathrm{CD}_{11 \mathrm{c}^{+} \mathrm{CD} 11 \mathrm{~b}^{+} \text {and }}$ $\mathrm{CD} 11 \mathrm{c}^{+} \mathrm{CD} 45 \mathrm{R} / \mathrm{B} 220^{+}$on day 5 and day 8 pi, respectively when compared with $P$. berghei ANKA-mono-infected mice (Figure 3).

The percentage of splenic DCs expressing TLR4, TLR9, MHC class II, and CD86 in the co-infected vs P. berghei ANKA-mono-infected mice was also compared. Both the proportion of CD $11 \mathrm{c}^{+} \mathrm{TLR}^{+}, \mathrm{CD} 11 \mathrm{c}^{+} \mathrm{TLR}^{+}$,


post-P. berghei ANKA infection, peaking on day 5 pi and then declining on day $8 \mathrm{pi}$. The percentage of CD11 $\mathrm{c}^{+}$

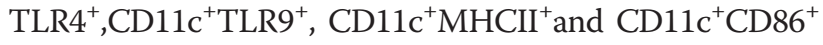
were both significantly lower in the co-infected mice on day 5 and day 8 pi when compared with P. berghei ANKAmono-infected mice (Figure 3 ). In addition, MFI of TLR4, TLR9, MHC class II, and CD86, which were expressed on


shown on Figure 3.

The kinetics of Tregs in both groups of mice was followed by flow cytometry to evaluate the role of Tregs in the response to malaria infection and to compare of the proportion/absolute number of Tregs between co-infected and $P$. berghei ANKA-monoinfected mice. Tregs in the spleen $\mathrm{CD} 4^{+} \mathrm{T}$-cell population increased after $P$. berghei ANKA infection with peaks appearing on day 5 pi and then declining. Also, the proportion/absolute number of Tregs on day 5 pi and day 8 pi was significantly higher in the co-infected mice than in the $P$. berghei ANKA-mono-infected mice (Figure 4).

Cytokine-secreting-Tregs in co-infected and $P$. berghei ANKA-mono-infected mice were also compared. The proportion/absolute number of IL-10-secreting-Tregs was slightly higher on day $5 \mathrm{pi}$, but then slightly lower on day 8 pi in the co-infected mice than in the $P$. berghei ANKA-mono-infected mice, however these differences were not significant. The proportion/absolute number of IFN- $\gamma$-secreting Tregs was significantly lower in co-infected mice than in P. berghei ANKAmono-infected mice on day 5 pi and day 8 pi (Figure 4).

Finally, the IFN- $\gamma$ and IL-10 produced by the Foxp3$\mathrm{CD}^{+}{ }^{+} \mathrm{T}$ cells in co-infected and P. berghei ANKA-monoinfected mice were compared. There was a significantly higher level of IFN $-\gamma^{+}$Foxp $3^{-} \mathrm{CD} 4^{+}$in the co-infected mice when compared with $P$. berghei ANKA-monoinfected mice, while the level of $\mathrm{IL}_{-} 10^{+} \mathrm{Foxp} 3^{-} \mathrm{CD} 4^{+}$ remained unchanged in both groups (Figure 5). 

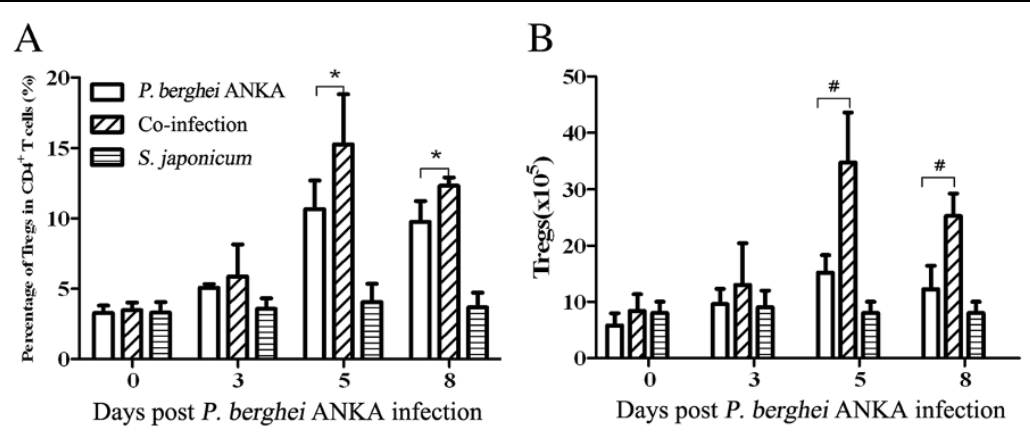

C

D
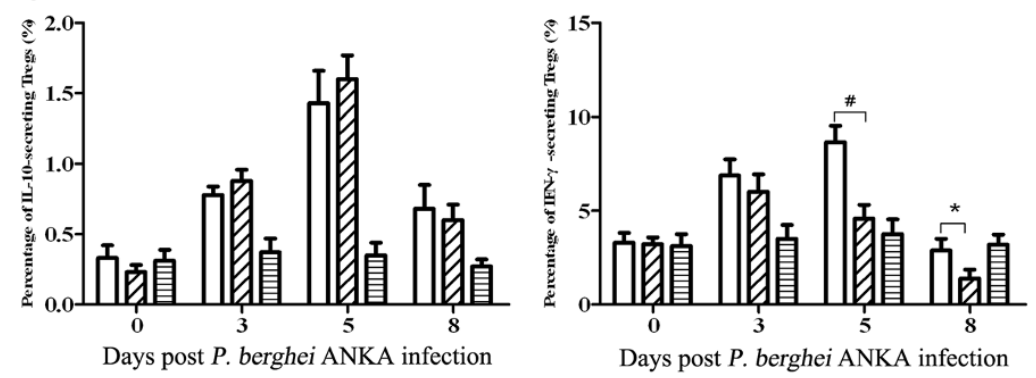

$\mathrm{E}$

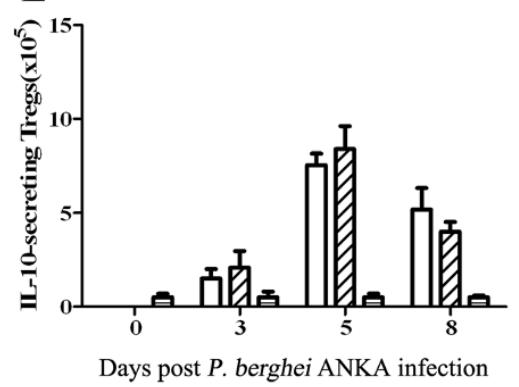

$\mathrm{F}$

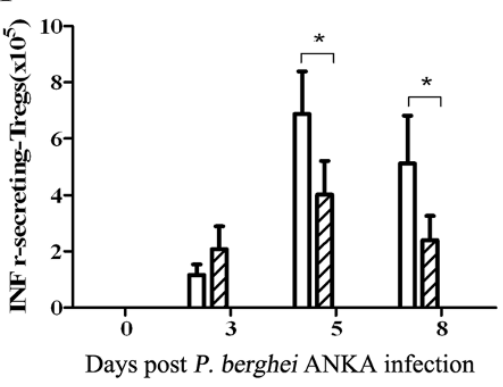

G


$\mathrm{H}$
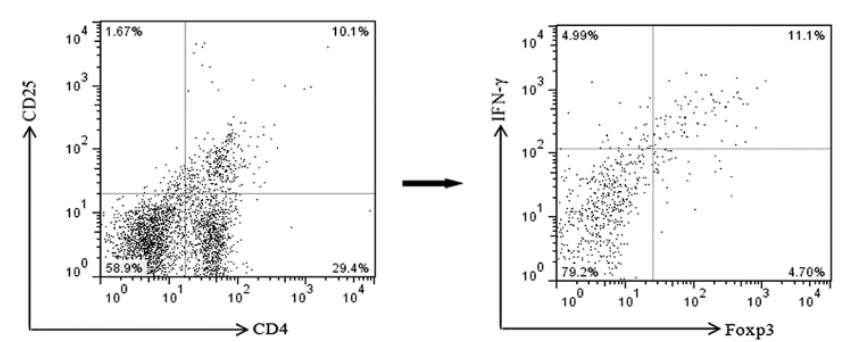

Figure 4 (See legend on next page.) 
(See figure on previous page.)

Figure 4 Mice in Plasmodium berghei ANKA-mono-infection group and Schistosoma japonicum-P. berghei ANKA-co-infection group were compared at different time points according to data obtained by flow cytometry. Proportion and absolute number of CD $4^{+}$ CD25 ${ }^{+}$Foxp3 ${ }^{+}$Tregs, IL-10-secreting Tregs, and IFN- $\gamma$-secreting Tregs were analysed in A, B, C, D, E, F, respectively. Gating strategy for identifying splenic Tregs were shown on $\mathbf{G}$ and $\mathbf{H}$ by representative dot plots. At each time point, at least three mice per group were sacrificed. Bars represent the mean values \pm SD. Student $t$ test by comparisons between the two groups was performed with * indicating $P<0.05$ and \# indicating $P<0.01$

\section{Histopathology}

Histopathology sections from co-infected and P. berghei ANKA-mono-infected mice, that had been euthanized on day 6,7 , and 8 post-infection, were analysed to evaluate the effect of pre-existing $S$. japonicum infection on P. berghei ANKA induced brain histology. Two normal animals were also euthanized to serve as a control. Conspicuous haemorrhage and mononuclear cell accumulation was observed in all of the P. berghei ANKAmono-infected mice (Figure 6A). In contrast, most of the co-infected mice exhibited no signs of brain pathology (similar to the normal controls). The brain vessel wall was intact and no immune cells were seen beside the vessel (Figure 6B). Mild vessel wall oedema was observed in two co-infected mice (Figure 6C).

\section{Discussion}

Many studies have been carried out to examine the impact of helminths on malaria infection, both in mouse models and in humans, yet the results are limited and often conflicting. Immune responses to parasitic infections are complex with many cytokines potentially playing crucial roles in the Th1-like or Th2-like responses. It is well known that helminth infections can ameliorate pro-inflammatory conditions, partly due to an inherent ability to induce Th2 responses [8-10]. In the current study, pre-existing $S$. japonicum infection enhanced Th2 responses to malaria infection, as evidenced by higher levels of IL-4, IL- 5 and IL-13 that were present in the co-infected mice. Also, the co- infected mice had lower levels of IFN- $\gamma$, which indicates a suppressed Th1 response to malaria infection. These cytokine changes may suggest a polarized Th2 response to malaria infection when concomitant with S. japonicum. The histopathology findings supported this point as well since no changes, or only mild brain pathology, developed in the co-infected mice.

It is generally considered that Tregs are involved in the regulation of the immune response to malaria infections while the potential to determine disease outcome remains unclear. A previous report demonstrated that Tregs were required to limit pro-inflammatory immune responses in $\mathrm{BALB} / \mathrm{c}$ mice in order to prevent ECM during secondary infections [24]. It has also been reported that Tregs can inhibit the Th1 immune response via modifying DCs and inducing the production of IL-10 during $P$. yoelii $17 \mathrm{XL}$ infection [25,26]. It has also been confirmed that the occurrence of Tregs during $P$. berghei ANKA infection is negatively associated with the production of IFN- $\gamma$ [22]. Induced and/or activated Tregs may be beneficial to the host in malaria (when parasitaemia is well-controlled) by down-regulating the inflammatory response and thereby preventing immunemediated pathology. On the other hand, Tregs could hamper the responses required for initial control of parasitaemia, permitting unbridled parasite growth if Tregs exerts its suppressive effects too soon, leading to severe disease [27]. In the current study, up-regulation of Tregs in the co-infected mice, indicated a preexisting S. japonicum infection had strengthened the
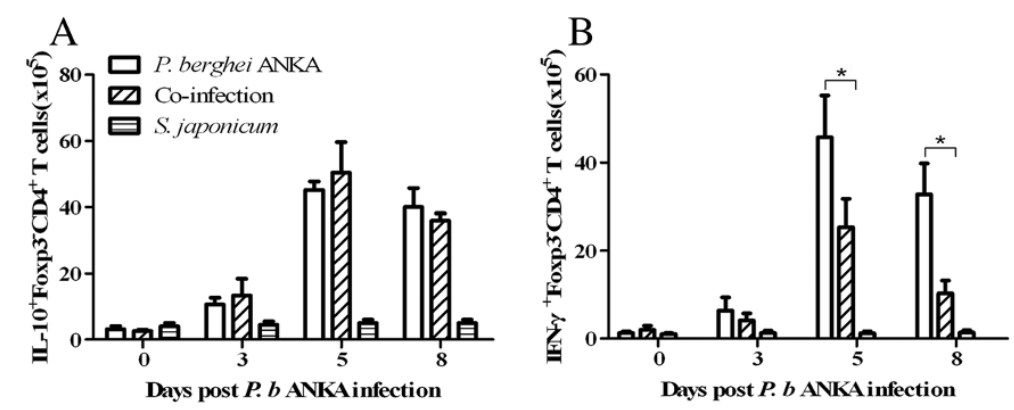

Figure 5 Absolute number of IL- $10^{+} \mathrm{Foxp}^{-} \mathrm{CD} 4^{+} \mathrm{T}$ cells (A) and IFN- $\gamma^{+}$Foxp $3^{-} \mathrm{CD} 4^{+} \mathrm{T}$ cells (B) were compared between the Plasmodium berghei ANKA-mono-infection group and Schistosoma japonicum-P. berghei ANKA-co-infection group at different time points. At each time point, at least three mice per group were sacrificed. Bars represent the mean values \pm SD. Student $t$ test by comparisons between the two groups was performed with * indicating $P<0.01$. 

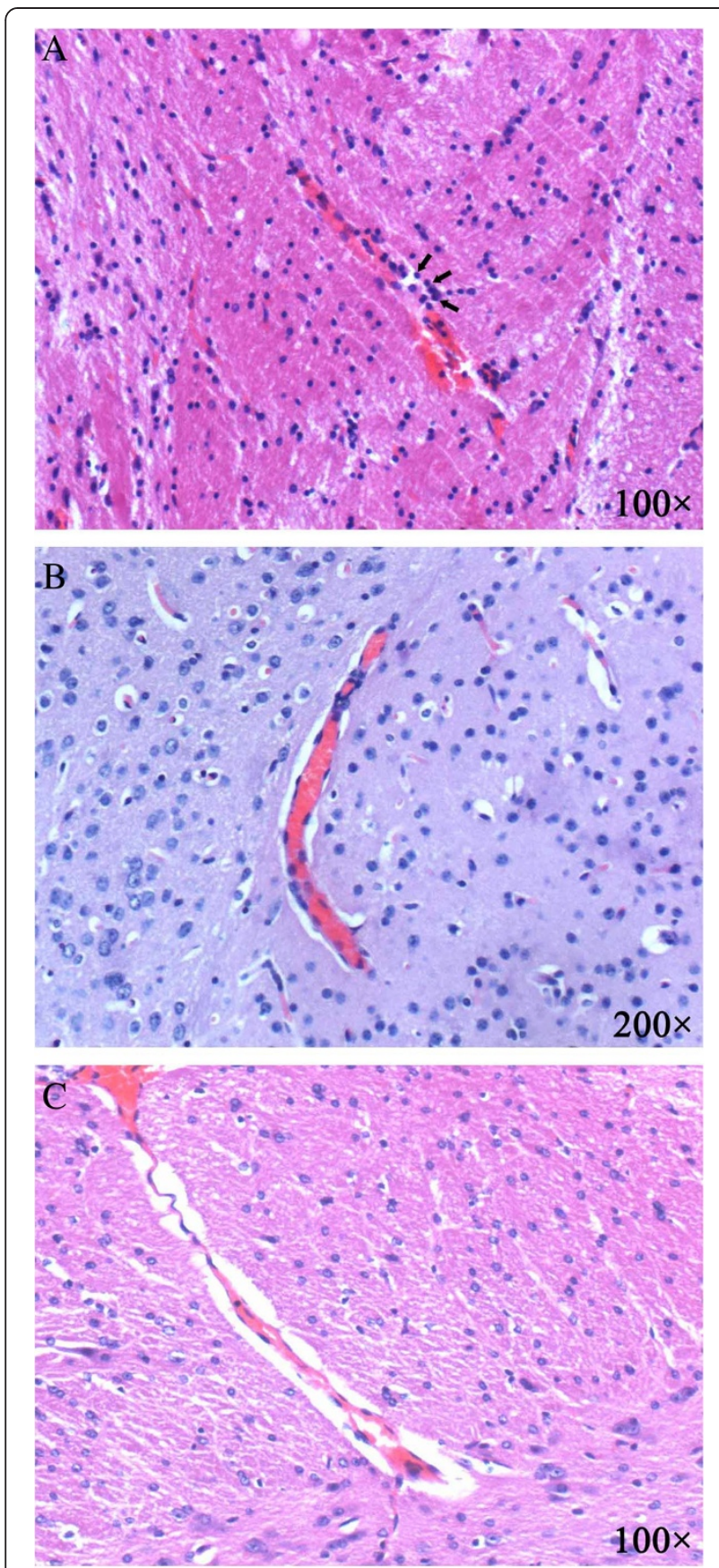

Figure 6 Brain sections were obtained from Plasmodium berghei ANKA-mono-infected and Schistosoma japonicum-P. berghei ANKA-co-infected mice on day 8 post- $P$. berghei ANKA infection. Conspicuous haemorrhage and mononuclear cell accumulation was observed in the P.b ANKA-mono-infected mice (A). In contrast, the co-infected mice exhibited intact brain vessels (B) or only mild vessel wall oedema (C). Sections stained with H\&E.

Th2 responses to malaria infection; this may protect against immunopathological impairment caused by Th1 responses. It is possible that the increased Tregs in the co-infected mice caused an enhanced Th2 response, which impeded the clearance of protozoan since significantly higher malaria parasitaemia (on day 7 and 8 post- $P$. berghei ANKA infection) existed in the coinfected mice and there was no significant difference in survival between S. japonicum-P. berghei ANKA-coinfected and $P$. berghei ANKA-mono-infected mice on day 6 - day 8 pi. Thus, it appears that there was a balance between both positive and negative effects, on the malaria infection, due to the increased Tregs.

Immune activity of Tregs can be mediated by a cellcontact-dependent mechanism and through the secretion of suppressive cytokines such as IL-10 [28,29] and promoting cytokines such as IFN- $\gamma$. It has been previously demonstrated that the proportion of IL-10-secreting Tregs is consistent with the Tregs population in $P . y$ infected mice and that the immune-suppressive activity of Tregs can be mediated through the secretion of IL-10 [25]. In the current study, the lower level of IFN- $\gamma$-secreting Tregs found in the co-infected mice indicated a weakened Th1 response to malaria infection. As the majority of the IL-10/ IFN- $\gamma$-producing T cells appear to be Foxp3 ${ }^{-}$, and thus constitute inducible Treg cells, the level of $\mathrm{IL}-10^{+} / \mathrm{IFN}-\gamma^{+}$-Foxp $3^{-} \mathrm{CD} 4^{+}$was further analysed and these results also indicated a suppressed Th1 response in the co-infected mice.

Recent studies have indicated that helminths also have direct anti-inflammatory effects on innate immune responses. Kane et al. [10] confirmed that the eggs of $S$. mansoni could suppress LPS-induced activation of immature murine DCs, including MHC class II. As specialized antigen-presenting cells (APCs), DCs play an important role in the activation of $\mathrm{T}$ cells and consequently in the induction of adaptive immune responses [30]. These cells are classified into two main subpopulations [31]: myeloid DCs $\left(\mathrm{CD} 11 \mathrm{c}^{+} \mathrm{CD} 11 \mathrm{~b}^{+}\right)$and plasmacytoid DCs $\left(\mathrm{CD} 11 \mathrm{c}^{+} \mathrm{CD} 45 \mathrm{R} / \mathrm{B} 220^{+}\right)$. Various patterns of proliferation may be manifested by these DCs when stimulated by different pathogens. TLRs are expressed on or within innate immune cells, including DCs, and recognize pathogen-associated molecular patterns from different microorganisms [32]. Accumulating evidence suggests that TLRs are also involved in immune responses to protozoan parasites [33-35]. It has been reported that TLR9 responds to haemozoin [33,34] and that TLR4 responds to the putative toxin glycosylphosphatidylinisitol (GPI) from Plasmodium falciparum [36], resulting in the production of cytokines and chemokines, as well as up-regulation of co-stimulatory molecules [33]. Polymorphisms in TLR9 and TLR4 are associated with disease manifestation [37,38]. Stimulation of T-cell responses and more importantly, induction of Th1/Th2 cell development, is associated with maturation of DCs as well as production of Th1/Th2 
cytokines [39-41]. Up-regulation of MHC class II and co-stimulatory molecules (i e CD80, CD86) are characteristic of the maturation of DCs [39-41]. High expression of MHC class II molecules is crucial for DCs to present antigens to $\mathrm{CD}^{+}$Th cells. A previous report has confirmed that the bulk of Th1/Th2 responses are present when the CD80/CD86 signaling pathway is blocked [42].

Ing et al. [43] demonstrated that DCs selectively phagocytose Plasmodium- pRBCs and present pRBCderived antigens to $\mathrm{CD} 4^{+} \mathrm{T}$ cells in vitro, suggesting that DCs may play a primary role as APCs in malaria infections. It has been shown that DCs are involved in the establishment and regulation of T-cell-mediated immunity in mice infected with malaria [20] and that blood-stage $P$. yoelii 17XL infection induced increased numbers of splenic $\mathrm{CD} 11 \mathrm{c}^{+} \mathrm{DCs}$ positive for MHC class II, CD80 and CD86, which is consistent with the establishment of the Th1 immune response [20]. In the current study, the effect of pre-existing S. japonicum infection on malaria infection was further evaluated by analysing distinct DC responses. Results indicated that pre-existing $S$. japonicum infection had an anti-inflammatory effect during the following malaria infection by directly suppressing the ability of DCs to produce pro-inflammatory mediators.

Bucher et al. [5] and Waknine-Grinberg et al. [4] found that a concomitant S. mansoni infection conferred protection against brain pathology caused by ECM, which is consistent with results from the current study. Tregs and the associated cytokines were further examined in the current study to investigate the immunomodulatory mechanism involved in the process of co-infections since these cytokines are important for establishing Th2 responses. In addition, assessment of DC responses during coinfection facilitated a better understanding of the direct anti-inflammatory effects a pre-existing schistosome infection has on the innate immune responses to malaria infection.

\section{Study limitations}

This was an acute study, conducted over an eight-day period, in mice that had been exposed to plasmodium infection after being infected eight weeks prior with $S$. japonicum. This means that the immune responses to $S$. japonicum would the strongest, before modulating to the chronic phase around week 12 post-infection.

\section{Conclusions}

The immune responses to malaria infection in P. berghei ANKA-S. japonicum co-infected mice were assessed and the changes in the cytokines, caused by pre-existing S. japonicum infection, were analysed. A Tregs-associated Th2 response played an important role in protecting ECM pathology. Pre-existing S. japonicum infection suppressed TLR ligand-induced DC maturation and had anti-inflammatory effects during malaria infection, not only by virtue of its ability to induce Th2 responses, but also by directly suppressing the ability of DCs to produce pro-inflammatory mediators.

\section{Abbreviations}

CM: Cerebral malaria; DCs: Dendritic cells; ECM: Experimental cerebral malaria; GPI: Glycosylphosphatidylinisitol; IFNy: Interferon-y; IL-10: Interleukin-10; mAb: Mono-clone antibody; pRBCs: Parasitized red blood cells; $P$. berghei ANKA: Plasmodium berghei ANKA; RT: Room temperature; TLRs: Toll-like receptors; Tregs: Regulatory $T$ cells.

\section{Competing interests}

The authors declare that they have no competing interests.

\section{Authors' contributions}

All of the authors collaborated on the work presented in this study. M-IW, Y$\mathrm{mC}$ and $\mathrm{E}-\mathrm{jL}$ defined the research theme; M-IW prepared the infected animal models, designed the methods, performed the experiments, and interpreted the results; $Y Z$ and $Y-j G$ analysed the data; and $M-I W, Y Z, E-j L$ and $Y-m C$ drafted the manuscript. All authors read and approved the final version of the manuscript.

\section{Acknowledgements}

Deepest thanks to Dr Motomi Torii (Ehime University Graduate School of Medicine, Ehime, Japan) for providing malarial parasite strains of $P$. berghei ANKA. This work was supported by grant from a scientific research project (No. L2012287) sponsored by the Education Department of Liaoning Province, China, grant from Natural Science Foundation of Liaoning Province (No. 2013021010). We thank LetPub for its linguistic assistance during the preparation of this manuscript.

\section{Author details}

${ }^{1}$ Department of Microbiology and Parasitology, College of Basic Medical Sciences, China Medical University, No. 92 Beier Road, Heping District, Shenyang 110001, China. ${ }^{2}$ Department of Immunology, College of Basic Medical Sciences, China Medical University, No. 92 Beier Road, Heping District, Shenyang 110001, China. ${ }^{3}$ Department of Sonography, Shengjing Hospital of China Medical University, No. 36 Sanhao Street, Heping District, Shenyang 110004, China.

Received: 22 May 2013 Accepted: 18 August 2013

Published: 14 September 2013

\section{References}

1. Legesse M, Erko B, Balcha F: Increased parasitemia and delayed parasite clearance in Schistosoma mansoni and Plasmodium berghei co-infected mice. Acta Trop 2004, 91:161-166.

2. Pierce SK, Miller LH: What malaria knows about the immune system that immunologists still do not? J Immunol 2009, 182:5171-5177.

3. Hunt NH, Grau GE: Cytokines: accelerators and brakes in the pathogenesis of cerebral malaria. Trends Immunol 2003, 24:491-499.

4. Waknine-Grinberg JH, Gold D, Ohayon A, Flescher E, Heyfets A, Doenhoff MJ, Schramm G, Haas H, Golenser J: Schistosoma mansoni infection reduces the incidence of murine cerebral malaria. Malar J 2010, 9:5.

5. Bucher K, Dietz K, Lackner P, Pasche B, Fendel R, Mordmüller B, Ben-Smith A, Hoffmann WH: Schistosoma co-infection protects against brain pathology but does not prevent severe disease and death in a murine model of cerebral malaria. Int J Parasitol 2011, 41:21-31.

6. Pierrot C, Wilson S, Lallet H, Lafitte S, Jones FM, Daher W, Capron M, Dunne DW, Khalife J: Identification of a novel antigen of Schistosoma mansoni shared with Plasmodium falciparum and evaluation of different crossreactive antibody subclasses induced by human schistosomiasis and malaria. Infect Immun 2006, 74:3347-3354.

7. Hu W, Yan Q, Shen DK, Liu F, Zhu ZD, Song HD, Xu XR, Wang ZJ, Rong YP, Zeng LC, Wu J, Zhang X, Wang JJ, Xu XN, Wang SY, Fu G, Zhang XL, Wang ZQ, Brindley PJ, McManus DP, Xue CL, Feng Z, Chen Z, Han ZG: Evolutionary and biomedical implications of a Schistosoma japonicum complementary DNA resource. Nat Genet 2003, 35:139-147. 
8. Pearce EJ, MacDonald AS: The immunobiology of schistosomiasis. Nat Rev Immunol 2002, 2:499-511.

9. Dunne DW, Cooke A: A worm's eye of the immune system: consequences for evolution of human autoimmune disease. Nat Rev Immunol 2005, 5:420-426.

10. Kane CM, Cervi L, Sun J, McKee AS, Masek KS, Shapira S, Hunter CA, Pearce EJ: Helminth antigens modulate TLR-initiated dendritic cell activation. J Immunol 2004, 173:7454-7461.

11. Nacher M: Interactions between worms and malaria: good worms or bad worms? Malar J 2011, 10:259.

12. Sokhna C, Le Hesran JY, Mbaye PA, Akiana J, Camara P, Diop M, Ly A, Druilhe P: Increase of malaria attacks among children presenting concomitant infection by Schistosoma mansoni in Senegal. Malar J 2004, 3:43.

13. Briand V, Watier L, LEH JY, Garcia A, Cot M: Coinfection with Plasmodium falciparum and Schistosoma haematobium: protective effect of schistosomiasis on malaria in Senegalese children? Am J Trop Med Hyg 2005, 72:702-707.

14. Lyke KE, Dicko A, Dabo A, Sangare L, Kone A, Coulibaly D, Guindo A, Traore K, Daou M, Diarra I, Plowe CV, Doumbo OK, Sztein MB: Association of Schistosoma haematobium infection with protection against acute Plasmodium falciparum malaria in Malian children. Infect Immun 2006 74:5718-5724

15. Lwin M, Last C, Targett GA, Doenhoff MJ: Infection of mice concurrently with Schistosoma mansoni and rodent malarias: contrasting effects of patent $S$. mansoni infections on Plasmodium chabaudi, $P$. yoelii and $P$. berghei. Ann Trop Med Parasitol 1982, 76:265-273.

16. Graham AL, Lamb TJ, Read AF, Allen JE: Malaria-filaria coinfection in mice makes malarial disease more severe unless filarial infection achieves patency. J Infect Dis 2005, 191:410-421.

17. Fernandez RD, Dubben B, Saeftel M, Endl E, Deininger S, Hoerauf A, Specht $S$ : Filarial infection induces protection against $P$. berghei liver stages in mice. Microbes Infect 2009, 11:172-180.

18. Wilson MS, Mentink-Kane MM, Pesce JT, Ramalingam TR, Thompson R, Wynn TA: Immunopathology of schistosomiasis. Immunol Cell Biol 2007, 85:148-154

19. Olds GR, Olveda R, Wu G, Wiest P, McGarvey S, Aligui G, Zhang S, Ramirez B, Daniel B, Peters P, Romulo R, Fevidal P, Tiu W, Yuan J, Domingo E, Blas B: Immunity and morbidity in schistosomiasis japonicum infection. Am J Trop Med Hyg 1996, 55(5 Suppl):121-126.

20. Zheng W, Wang $\mathrm{QH}$, Liu J, Feng $\mathrm{H}$, Wu JJ, Cao YM: Distinct host-related dendritic cell responses during the early stage of Plasmodium yoelii infection in susceptible and resistant mice. Parasite Immunol 2010, 32:324-334

21. Smithers SR, Terry RJ: The infection of laboratory hosts with cercariae of Schistosoma mansoni and the recovery of the adult worms. Parasitology 1965, 55:695-700.

22. Wu JJ, Chen G, Liu J, Wang T, Zheng W, Cao YM: Natural regulatory T cells mediate the development of cerebral malaria by modifying the pro-inflammatory response. Parasitol Int 2010, 59:232-241.

23. Ma SH, Zheng L, Liu YJ, Guo SY, Feng H, Chen G, Li DM, Wang JC, Cao YM: Plasmodium yoelii: influence of antimalarial treatment on acquisition of immunity in BALB/C and DAB/2 mice. Exp Parasitol 2007, 116:266-272.

24. Nie CQ, Bernard NJ, Schofield $L$, Hansen DS: CD4+CD25+ regulatory $T$ cells suppress CD4+ T-cell function and inhibit the development of Plasmodium berghei-specific TH1 responses involved in cerebral malaria pathogenesis. Infect Immun 2007, 75:2275-2282

25. Chen G, Liu J, Wang QH, Wu Y, Feng H, Zheng W, Guo SY, Li DM, Wang JC, Cao YM: Effects of CD4(+)CD25(+)Foxp3(+)regulatory T cells on early Plasmodium yoelii 17XL infection in BALB/c mice. Parasitology 2009, 136:1107-1120

26. Zheng W, Wang QH, Feng H, Liu J, Meng HR, Cao YM: CD4+ CD25+ Foxp3+ regulatory $T$ cells prevent the development of Th1 immune response by inhibition of DC function during the early stage of Plasmodium yoelii infection in susceptible BALB/c mice. Folia Parasitol (Praha) 2009, 56:242-250.

27. Walther M, Tongren JE, Andrews L, Korbel D, King E, Fletcher H, Andersen RF, Bejon P, Thompson F, Dunachie SJ, Edele F, de Souza JB, Sinden RE, Gilbert SC, Riley EM, Hill AV: Upregulation of TGF-beta, FOXP3, and CD4+ CD25+ regulatory $T$ cells correlates with more rapid parasite growth in human malaria infection. Immunity 2005, 23:287-296.

28. Couper KN, Blount DG, Riley EM: IL-10: the master regulator of immunity to infection. J Immunol 2008, 180:5771-5777.
29. Nakamura K, Kitani A, Strober W: Cell contact-dependent immunesuppression by CD4+CD25+regulatory $T$ cells is mediated by cell surface-bound transforming growth factor beta. J Exp Med 2001, 194:629-644.

30. Diao J, Winter E, Chen W, Cantin C, Cattral MS: Characterization of distinct conventional and plamacytoid dendritic cell-committed precursors in murine bone marrow. J Immunol 2004, 173:1826-1833.

31. Gilliet $\mathrm{M}$, Boonstra A, Paturel $\mathrm{C}$, Antonenko $\mathrm{S}, \mathrm{Xu} X \mathrm{~L}$, Trinchieri G, O'Garra A, Liu YJ: The development of murine plasmacytoid dendritic cell precursors is differentially regulated by FLT3-ligand and granulocyte / macrophage colony-stimulating factor. J Exp Med 2002, 195:953-958.

32. Iwasaki A, Medzhitov R: Toll-like receptor control of the adaptive immune responses. Nat Immunol 2004, 5:987-995.

33. Coban C, Ishii KJ, Kawai T, Hemmi H, Sato S, Uematsu S, Yamamoto M, Takeuchi O, Itagaki S, Kumar N, Horii T, Akira S: Toll-like receptor 9 mediates innate immune activation by the malaria pigment hemozoin. J Exp Med 2005, 201:19-25.

34. Hisaeda H, Tetsutani K, Imai T, Moriya C, Tu L, Hamano S, Duan X, Chou B, Ishida H, Aramaki A, Shen J, Ishii KJ, Coban C, Akira S, Takeda K, Yasutomo K, Torii M, Himeno K: Malaria parasites require TLR9 signaling for immune evasion by activating regulatory T cells. J Immunol 2008, 180:2496-2503.

35. Akira S, Takeda K, Kaisho T: Toll-like receptors: critical proteins linking innate and acquired immunity. Nat Immunol 2001, 2:675-680.

36. Krishnegowda G, Hajjar AM, Zhu J, Douglass EJ, Uematsu S, Akira S, Woods AS, Gowda DC: Induction of proinflammatory responses in macrophages by the glycosylphosphatidylinisitols (GPIs) of Plasmodium falciparum: cell signalling receptors, GPI structural requirements, and regulation of GPI activity. J Biol Chem 2005, 280:8606-8616.

37. Mockenhaupt FP, Cramer JP, Hamann L, Stegemann MS, Eckert J, Oh NR, Otchwemah RN, Dietz E, Ehrhardt S, Schröder NW, Bienzle U, Schumann RR: Toll-like receptor (TLR) polymorphisms in African children: common TLR-4 variants predispose to severe malaria. J Commun Dis 2006, 38:230-245.

38. Mockenhaupt FP, Hamann L, von Gaertner C, Bedu-Addo G, von Kleinsorgen C, Schumann RR, Bienzle U: Common polymorphisms of toll-like receptors 4 and 9 are associated with the clinical manifestation of malaria during pregnancy. J Infect Dis 2006, 194:184-188.

39. Ing R, Stevenson MM: Dendritic cell and NK cell reciprocal cross talk promotes gamma interferon-dependent immunity to blood-stage Plasmodium chabaudi AS infection in mice. Infect Immun 2009, 77:770-782.

40. Wykes MN, Liu XQ, Beattie L, Stanisic DI, Stacey KJ, Smyth MJ, Thomas R, Good MF: Plasmodium strain determines dendritic cell function essential for survival from malaria. PLoS Pathog 2007, 3:e96.

41. Stevenson MM, Urban BC: Antigen presentation and dendritic cell biology in malaria. Parasite Immunol 2006, 28:5-14.

42. Taylor-Robinson AW, Smith EC: Modulation of experimental blood stage malaria through blockade of the B7/CD28 T-cell costimulatory pathway. Immunology 1999, 96:498-504.

43. Ing R, Segura M, Thawani N, Tam M, Stevenson MM: Interaction of mouse dendritic cells and malaria-infected erythrocytes: uptake, maturation, and antigen presentation. J Immunol 2006, 176:441-450.

doi:10.1186/1475-2875-12-322

Cite this article as: Wang et al:: Pre-existing Schistosoma japonicum infection alters the immune response to Plasmodium berghei infection in C57BL/6 mice. Malaria Journal 2013 12:322.

\section{Submit your next manuscript to BioMed Central and take full advantage of:}

- Convenient online submission

- Thorough peer review

- No space constraints or color figure charges

- Immediate publication on acceptance

- Inclusion in PubMed, CAS, Scopus and Google Scholar

- Research which is freely available for redistribution 